\title{
London Deauville Criteria Point Scale X
}

National Cancer Institute

\section{Source}

National Cancer Institute. London Deauville Criteria Point Scale X. NCI Thesaurus. Code C136879.

New areas of uptake unlikely to be related to lymphoma. 